科 学通报

\title{
$\mathrm{C}_{60}$ 与亲核试剂的反应 ${ }^{*}$
}

黄祖恩 孙雨龙 蔡瑞芳

(复旦大学化学系, 上海200433)

\section{关镬词 $\mathrm{C}_{60}$ 、亲校试剂、反应}

Kroto 等 ${ }^{[1]} 1985$ 年发现 $\mathrm{C}_{60}$ 并提出 $\mathrm{C}_{60}$ 笼形结构模型, 但对 $\mathrm{C}_{60}$ 进行大量深入的研究是在 Krätschmer 等人 ${ }^{[2]}$ 报道了制备克级 $\mathrm{C}_{60}$ 方法之后迅速开展的. 由于 $\mathrm{C}_{60}$ 中每个碳原子所处的物 理环境相同, 因此在对 $\mathrm{C}_{60}$ 进行化学修饰的过程中, 控制修饰基团的数目及其在 $\mathrm{C}_{60}$ 上的位置并 不容易. 尽管在 $\mathrm{C}_{60}$ 化学修饰方面已有不少工作, 但目前只报道了很少几个纯的 $\mathrm{C}_{60}$ 衍生物, 报 道的产物绝大多数是混合物, 如 Krusic 等 ${ }^{[3]}$ 利用光化学反应, 合成了含有多个烷基的 $\mathrm{C}_{60}$ 行生 物: $\mathrm{C}_{60}\left(\mathrm{CH}_{3}\right)_{n}(n=1-34)$ 和 $\mathrm{C}_{60}\left(\mathrm{CH}_{2} \mathrm{C}_{6} \mathrm{H}_{5}\right)_{n}(n=1-15)$; 我们曾在低价稀土碘化物 $\left(\mathrm{SmI}_{2}\right.$ 或 $\left.\mathrm{YbI}_{2}\right)$ 的存在下, 用 $\mathrm{C}_{60}$ 和卤代烃 $\left(\mathrm{C}_{3} \mathrm{H}_{5} \mathrm{Br}\right.$ 或 $\left.\mathrm{C}_{6} \mathrm{H}_{5} \mathrm{CH}_{2} \mathrm{Cl}\right)$ 反应, 得到 $\mathrm{C}_{60}\left(\mathrm{CH}_{2} \mathrm{CH}=\mathrm{CH}_{2}\right) n(n=$ $1-10)$ 和 $\mathrm{C}_{60}\left(\mathrm{CH}_{2} \mathrm{C}_{6} \mathrm{H}_{5}\right)_{n}(n=1-14)$. $\mathrm{C}_{60}$ 是一个贫电子分子, 有很高的电子亲合势 (2. $65 \mathrm{eV})^{[4]}$. Hirsch 等 ${ }^{[5]}$ 曾用叔丁基锂或乙基溴化镁滴定 $\mathrm{C}_{60}$, 制得 $\mathrm{t}-\mathrm{BuC}_{60} \mathrm{H}$ 和 $\mathrm{EtC}_{60} \mathrm{H}$. 本文 研究 $\mathrm{C}_{60}$ 与 2-甲基烯丙基氯化镁的反应, 通过控制反应条件, 合成了 $\mathrm{C}_{60} \mathrm{H}\left[\mathrm{CH}_{2} \mathrm{C}\left(\mathrm{CH}_{3}\right)=\mathrm{CH}_{2}\right]$ 和 $\mathrm{C}_{60} \mathrm{H}_{8}\left[\mathrm{CH}_{2} \mathrm{C}\left(\mathrm{CH}_{3}\right)=\mathrm{CH}_{2}\right]_{8}$. 此外, 还用狮基钠与 $\mathrm{C}_{60}$ 反应, 制得 $\mathrm{C}_{60} \mathrm{H}_{8}\left(\mathrm{C}_{9} \mathrm{H}_{7}\right)_{8}$.

\section{1 实 验 部 分}

\section{1 试剂及仪器}

使用的溶剂 (苯、乙醚、四氢呋喃)均经脱氧、脱水处理, 用前蒸出. 富勒烯采用石墨电极电 弧放电法制备, 中性氧化铝柱层析分离出纯 $\mathrm{C}_{60}$. 2-甲基烯丙基氯化镁由金属镁与 2-甲基烯 丙基氯反应制得. 金属钠与狮反应生成狮基钠.

产物合成水解前的操 作均在纯化的氮气中进行,所用的玻璃仪器为 Schlenk 型标准磨口 玻璃仪器. 紫外可见光谱用 UV-240岛津紫外分光光度计测定. 红外光谱用 Nicolet-FT-IR5DX 红外光谱仪测定, 采用溴化钾压片法. 高压液相色谱 (HPLC) 用 Shimadzu LC-4A 型高压 液相色谱仪测定. ${ }^{1}$ HNMR 谱用 Bruker MSL-300型核磁共振仪测定. 快原子轰击质谱 (FABMS) 用 VG-Quattro 四极质谱仪测定.

\section{2 化合物的合成}

(1) $\mathrm{C}_{60} \mathrm{H}\left[\mathrm{CH}_{2} \mathrm{C}\left(\mathrm{CH}_{3}\right)=\mathrm{CH}_{2}\right]$ (1) 的合成 $40 \mathrm{mgC}_{60}(0.0556 \mathrm{mmol})$ 溶于 $30 \mathrm{ml}$ 苯中, 剧 烈搅拌下缓慢滴入 $1.5 \mathrm{ml}$ 含 $0.071 \mathrm{mmol} 2$-甲基烯丙基氯化镁的四氢呋喃溶液, 滴加完毕后室 温搅拌 $1 \mathrm{~h}$,用稀盐酸水解, 棕红色有机相浓缩至干,得到棕红色固体 (1).

(2) $\mathrm{C}_{60} \mathrm{H}_{8}\left[\mathrm{CH}_{2} \mathrm{C}\left(\mathrm{CH}_{3}\right)=\mathrm{CH}_{2}\right]_{8}$ 的合成 $50 \mathrm{mgC}_{60}(0.0694 \mathrm{mmol})$ 置于柱形瓶中, 加入

1993-04-23收稿,1993-07-08收修改稿.

- 上海市科学技术委员会资助课题. 
$30 \mathrm{ml}$ 四氢呋喃, 搅拌下加入含 $1.08 \mathrm{mmol} 2$-甲基烯丙基氯化镁的四氢呋喃溶液, 搅拌反应一 周, 离心, 黑色溶液浓缩至干, 依次用苯、乙醚洗涤, 然后用稀盐酸水解, 处理得橙红色固体 (2).

(3) $\mathrm{C}_{60} \mathrm{H}_{8}\left(\mathrm{C}_{9} \mathrm{H}_{7}\right)_{8}$ 的合成 操作同 (2). $50 \mathrm{mgC}_{60}$ 与 $3.9 \mathrm{mmol}$ 狮基钠在四氢呋喃中反 应,离心所得黑色溶液浓缩至干, 依次用苯、乙瞇洗涤, 水解后处理得到棕色固体 (3).

\section{2 结 果与 讨 论}

产物的紫外可见光谱(图1)说明2-甲基烯丙基氯化镁或狮基钠与 $\mathrm{C}_{60}$ 反应生成了新的化合 物. 三个产物和 $\mathrm{C}_{60}$ 在 $430 \mathrm{~nm}$ 和 $600 \mathrm{~nm}$ 处都有特征吸收峰, 1 在 $405 \mathrm{~nm}$ 处与 $\mathrm{C}_{60}$ 有相同的吸收峰. $\mathrm{C}_{60}$ 在 $440-580 \mathrm{~nm}$ 的范围内

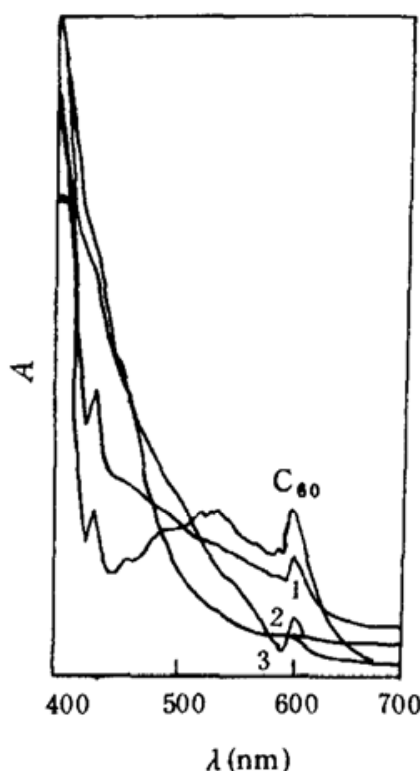

图1 $\mathrm{C}_{60}$ 及产物 $1,2,3$ 紫外可见 光谱

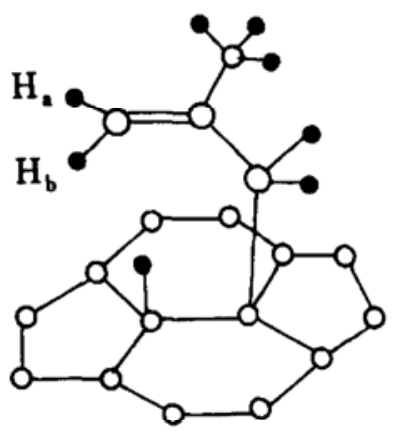

图2 $\mathrm{C}_{60} \mathrm{H}\left[\mathrm{CH}_{2}\left(\mathrm{CH}_{3}\right)=\mathrm{CH}_{2}\right]$ $\mathrm{O}$ 为 $\mathrm{C}$, $\mathrm{O}$ 为 $\mathrm{H}$ 有很宽吸收峰, 而产物的吸收峰在这个区间中都有不同程度的 变化. 与 $\mathrm{C}_{60}$ 吸收曲线相比, 2 和 3 在 $405 \mathrm{~nm}$ 处没有吸收峰, 2 在 $450 \mathrm{~nm}$ 处有一个新吸收峰.

1 的主要红外吸收峰有: $525(\mathrm{vs}), 578(\mathrm{~s}), 672(\mathrm{w}), 806$ (w), 837(w), 900 (s), 1181 (s),1371 (w),1428(s), 1459(w), $1541(w), 1638(w), 2844(w), 2918(w), 2959(w)$ 和 $3066(w)$ $\mathrm{cm}^{-1}$, 其 中除了 $\mathrm{C}_{60}$ 的红外特征 峰 ${ }^{[6]}: 525,578,1181$ 和 $1428 \mathrm{~cm}^{-1}$ 外, 还有甲基烯丙基的特征吸收峰: 1459, 1638, 2844,2959 和 $3066 \mathrm{~cm}^{-1}$. 2 的主要红外吸收峰有: $541(\mathrm{w}), 894$ (s), $1034(\mathrm{w}), 1375(\mathrm{~m}), 1441(\mathrm{~m}), 1644(\mathrm{~m}), 2853(\mathrm{w}), 2927$ (s), 2960 (w) 和 $3074(\mathrm{w}) \mathrm{cm}^{-1}$, 甲基烯丙基的特征峰相当显 著, 但 $\mathrm{C}_{60}$ 的特征峰全部消失, 说明 $\mathrm{C}_{60}$ 与多个2-甲基烯丙基键 合后, $\mathrm{C}_{60}$ 的振动模式发生了显著的变化. 3 的主要红外吸收峰 有: $528(\mathrm{~m}), 713(\mathrm{~s}), 766(\mathrm{vs}), 978(\mathrm{~m}), 1025(\mathrm{~m}), 1391(\mathrm{~m})$, $1459(\mathrm{~s}), 1606(\mathrm{~m}), 1713(\mathrm{~s}), 2853(\mathrm{~m}), 2927(\mathrm{~s}), 3017(\mathrm{w})$ 和 $3066(\mathrm{w}) \mathrm{cm}^{-1}$, 这里仅保留了 $\mathrm{C}_{60}$ 的一个特征峰 $\left(528 \mathrm{~cm}^{-1}\right)$, 狮 基的红外特征峰 $713,766,1025,1391,1459,1606,2927$ 和 $3066 \mathrm{~cm}^{-1}$ 均出现在 3 的红外光谱中.

研究1的高压液相色谱(实验条件: 硅胶 $\mathrm{Si} 60,5 \mu \mathrm{m}$, 柱 $25 \times$ $0.5 \mathrm{~cm}$, 淋洗液 $5 \%$ 甲苯/已烷溶液 $(V / V)$, 流速 $0.8 \mathrm{ml} \mathrm{min}^{-1}$, 检测波长 $330 \mathrm{~nm}$ ), 只出现二个峰, 第一个峰的出峰时间与 $\mathrm{C}_{60}$ 出现的时间相同,第二个峰与新化合物相应.

1 的 ${ }^{1} \mathrm{HNMR}$ 谱上有 5 个峰, 化学位移分别是 $6.72,5.68$, $5.52,4.21$ 和 $2.40 \mathrm{ppm}$, 峰面积之比为 $1: 1: 1: 2: 3$, 因此 HPLC 中第二个峰相对应的新化合物是 $\mathrm{C}_{60} \mathrm{H}\left[\mathrm{CH}_{2} \mathrm{C}\left(\mathrm{CH}_{3}\right)=\mathrm{CH}_{2}\right]$, 结构见图 2. 氢的具体归属为: $6.72 \mathrm{ppm}\left(\mathrm{s}, 1 \mathrm{H}: \mathrm{C}_{60}-\mathrm{H}\right), 5.68$ $\operatorname{ppm}\left(\mathrm{s}, 1 \mathrm{H}: \mathrm{H}_{\mathrm{a}}\right), 5.52 \mathrm{ppm}\left(\mathrm{s}, 1 \mathrm{H}: \mathrm{H}_{\mathrm{b}}\right), 4.21 \mathrm{ppm}\left(\mathrm{s}, 2 \mathrm{H}: \mathrm{CH}_{2}\right)$, 2. $40 \mathrm{ppm}\left(\mathrm{s}, 3 \mathrm{H}: \mathrm{CH}_{3}\right)$.

FAB-MS 分析2 (快原子轰击条件: 间硝基苠醇为底物), 碎片离子峰为 $m / Z: 721,775,831,887,942,998,1051,1108$ 和 1163 , 相应丰度比为 $182: 37: 14: 6: 4: 3: 2: 2: 1$, 呈依次递减序列. 基峰 $m / Z 721$ 对应于 $\mathrm{HC}_{60}^{+}$, 相 邻峰值差 $55 \mathrm{u}$ (一个甲基烯丙基质量数, 有些相邻峰差与 $55 \mathrm{u}$ 有出入, 可能是由于仪器漂移引 起), 这些离子峰对应于含有 1 到 8 个甲基烯丙基的 $\mathrm{C}_{60}$ 衍生物, 因此利用2-甲基烯丙 
基氯化镁与 $\mathrm{C}_{60}$ 反应能够形成 $\mathrm{C}_{60} \mathrm{H}_{8}\left[\mathrm{CH}_{2} \mathrm{C}\left(\mathrm{CH}_{3}\right)=\mathrm{CH}_{2}\right]_{8}$, 与 $\mathrm{C}_{60}$ 相连的有机基团的最大数目 要比自由基反应所得产物中与 $\mathrm{C}_{60}$ 相连的有机基团的最大数目要小得多. 尽管 $m / Z 721$ 丰度远 大于其它离子峰, 但 2 的 HPLC 显示 2 中只有极微量的 $\mathrm{C}_{60}$, 因此 $m / Z 721$ 的 $\mathrm{HC}_{60}^{+}$主要来源于 $\mathrm{C}_{60}$ 衍生物的碎裂, 低加成产物的离子峰来源于样品中低加成产物或来源于 $\mathrm{C}_{60} \mathrm{H}_{8}\left[\mathrm{CH}_{2} \mathrm{C}\left(\mathrm{CH}_{3}\right)=\right.$ $\left.\mathrm{CH}_{2}\right]_{8}$ 的裂解, 尚有待进一步研究.

3 的 FAB-MS 谱图中, 碎片离子峰为 $m / Z: 721,837,952,1068,1184,1300,1414,1528$ 和 1643 , 丰度比为: $54: 4: 4: 3: 2: 2: 2: 1: 1$, 依次递减, 与 2 相似, 有 1 到 8 个狮基接在 $\mathrm{C}_{60}$ 上, $\mathrm{C}_{60}$ 与狮 基钠反应生成 $\mathrm{C}_{60} \mathrm{H}_{8}\left(\mathrm{C}_{9} \mathrm{H}_{7}\right)_{8}$.

$\mathrm{C}_{60}$ 化学修饰方兴未艾. 本文对亲核试剂与 $\mathrm{C}_{60}$ 的加成反应进行了一些研究, 发现贫电子的 $\mathrm{C}_{60}$ 易与一些金属有机试剂反应,生成的产物比 $\mathrm{C}_{60}$ 与自由基反应得到的产物简单, 反应容易控 制. 这类反应将会成为 $\mathrm{C}_{60}$ 化学修饰的有效方法之一.

致谢复旦大学物理系李郁芬、钱士雄提供了电弧法制得的含富勒烯烟灰; 化学系吴世 晖、舒联合在 HPLC 测试方面给予很大帮助; 中国科学院上海有机化学研究所徐永贞等人进 行了 FAB-MS 测试, 在此一并致以谢意.

\section{参考文献}

[1] Kroto, H. W. , Heath, J. R. et al. , Nature, 1985,318:162.

[2] Krātschmer, W. , Lamb, L. D. et al. , Nature, 1990,347:354.

[3] Krusic, P. J., Wasserman, E. et al. , Science, 1991,254:1183.

[4] Wang, L. S., Conceicao, J. et al., Chem. Phys. Lett., 1991,182:5.

[5] Hirsch, A., Soi, A., Karfunkel, H. R., Angew. Chem. Int. Ed. Engl., 1992,31:766.

[6] Krätschmer, W. , Fostiropoulous, K., Huffman, D. R. , Chem. Phys. Lett., 1990,170:167. 